AROUEOLOGÍA Y SocIEDAD

№ 28, 2014: 321-334

ISSN: 0254-8062

\title{
DE LO CUANTITATIVO A LO CONDUCTUAL: APLICACIÓN DEL ÍNDICE BOONE EN EL ANÁLISIS DE DEPÓSITOS ESTRATIGRÁFICOS DE CHAVÍN DE HUÁNTAR
}

\author{
Christian Mesía Montenegro \\ MINISTERIO DE CULTURA \\ christian.mesia@gmail.com
}

\section{RESUMEN}

En este artículo se aplica el índice de diversidad Boone, en el estudio de 200 depósitos estratigráficos, distribuidos en cinco unidades espaciales prehistóricas, conteniendo más de 20000 materiales pertenecientes a los períodos Formativo Medio y Tardío, recuperados en el Sector Wacheqsa de Chavín de Huántar. La aplicación del índice Boone, cuyo efecto de tamaño de muestra fue controlado mediante rutinas Monte Carlo, permitió la identificación de variaciones cuantitativas en la distribución del registro arqueológico, las cuales reflejan variaciones conductuales en las actividades desarrolladas en las cinco unidades espaciales mencionadas.

Palabras Clave: Chavin de Huantar, Sector Wacheqsa, Periodo Formativo, Índice Boone, Rutina Montercalo.

\begin{abstract}
In this paper, the Boone Index is applied in the study of 200 strata, distributed among five spatial units, containing more than 20000 archaeological materials that belonged to the Middle and Late Formative periods in Chavin de Huantar's Wacheqsa Sector. The sample's size effect was controlled by using Monte Carlo routines, while the Index application allowed the identification of quantitative variations in the archaeological record's distribution, which in turn reflect distinctions in the activities that took place in the aforementioned spatial units.
\end{abstract}

KeYwoRDS: Chavin de Huantar, Wacheqsa sector, Formative Period, Boone Index, Monte Carlo routine.

\section{INTRODUCCIÓN}

El presente artículo da cuenta de la aplicación de una herramienta destinada a la identificación de variaciones conductuales expresadas cuantitativamente en la homogeneidad o heterogeneidad de materiales arqueológicos y conjuntos estratigráficos. Se utiliza el índice Boone (Boone 1987) el cual 
permite la cuantificación de índices de diversidad por clase de artefacto al interior de cada depósito. Cuando estos índices son agrupados en unidades espaciales, es posible identificar el comportamiento de los artefactos en cada unidad espacial. El índice se representa en la variable Hi, en donde elevados valores Hi indican homogeneidad en el registro arqueológico, mientras que índices Hi bajos indican heterogeneidad. La homogeneidad puede ser interpretada como un indicador de tareas y/o actividades que involucraron una variedad muy reducida de materiales arqueológicos, mientras que la heterogeneidad es un indicador de que dichas actividades involucraron una diversidad mayor de artefactos arqueológicos. El efecto del tamaño de muestra es resuelto mediante la aplicación de rutinas Monte Carlo de hasta 10000 repeticiones. Este índice se aplica a los más de 20000 materiales arqueológicos, recuperados en 200 depósitos estratigráficos, excavados en el Sector Wacheqsa del Centro ceremonial de Chavín de Huántar.

\section{El centro ceremonial de Chavín de Huántar}

El centro ceremonial de Chavín de Huántar está localizado en la provincia de Huari, departamento de Ancash, a 3200 msnm, en la intersección de los ríos Wacheqsa y Mosna (Fig. 1). Chavín de Huántar ha gozado de una posición privilegiada en relación a los debates referentes a complejidad social, destreza constructiva, simbolismo iconográfico y diversidad cultural, siendo un constante punto de referencia en la literatura arqueológica (Bennett 1944; Burger 1984, 1988, 1988; Kembel 2001, 2008; Kembel y Rick 2004; Lumbreras 1977, 1989, 1993, 2007; Rick 2005, 2006, 2008; Tello 1960). Las investigaciones se desarrollaron en el sector Wacheqsa (Mesia 2007, 2012), localizado inmediatamente al norte del área monumental (Fig. 2).

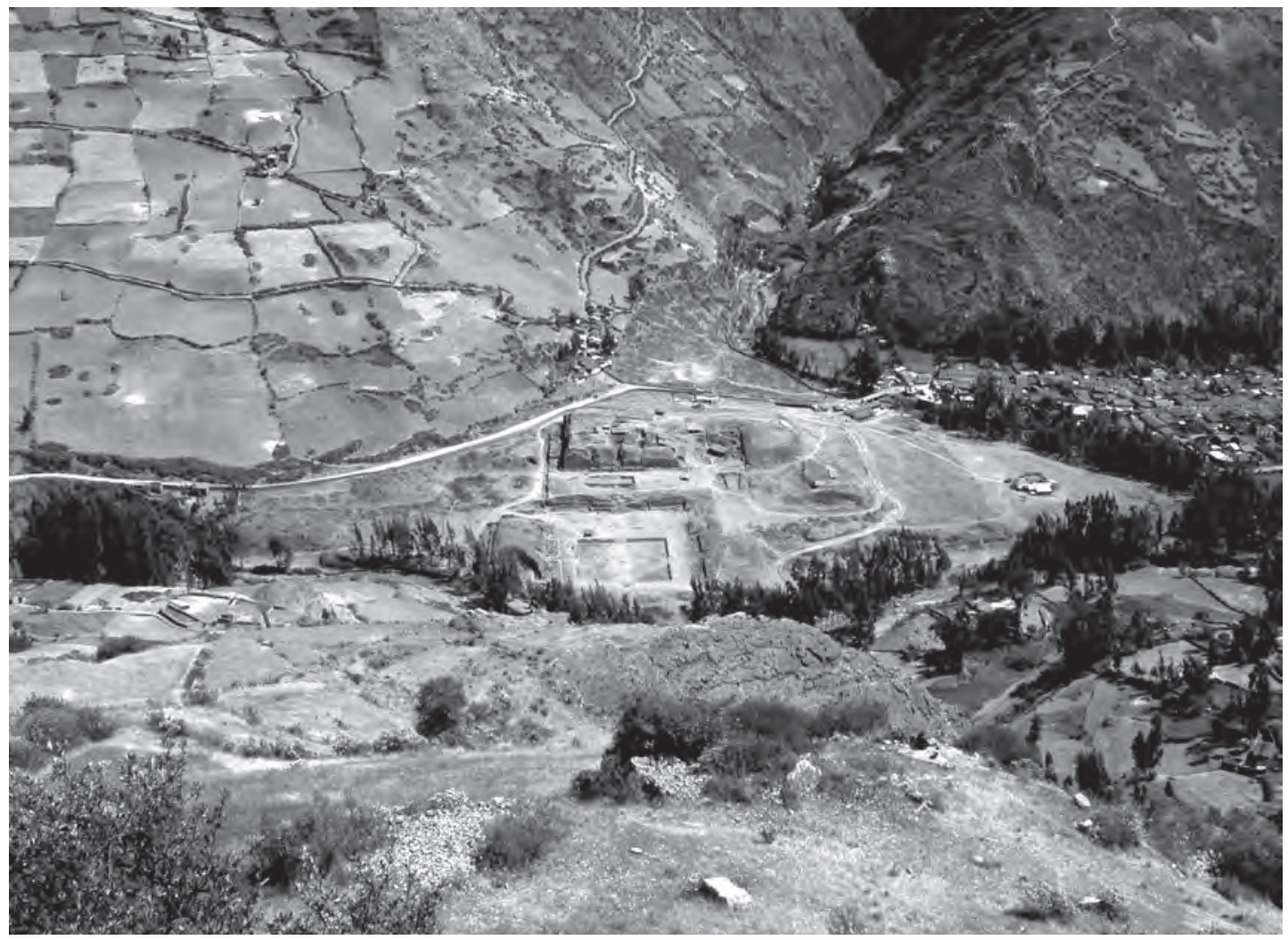

Figura 1. Vista de Chavín de Huántar desde la ribera este del río Mosna. 


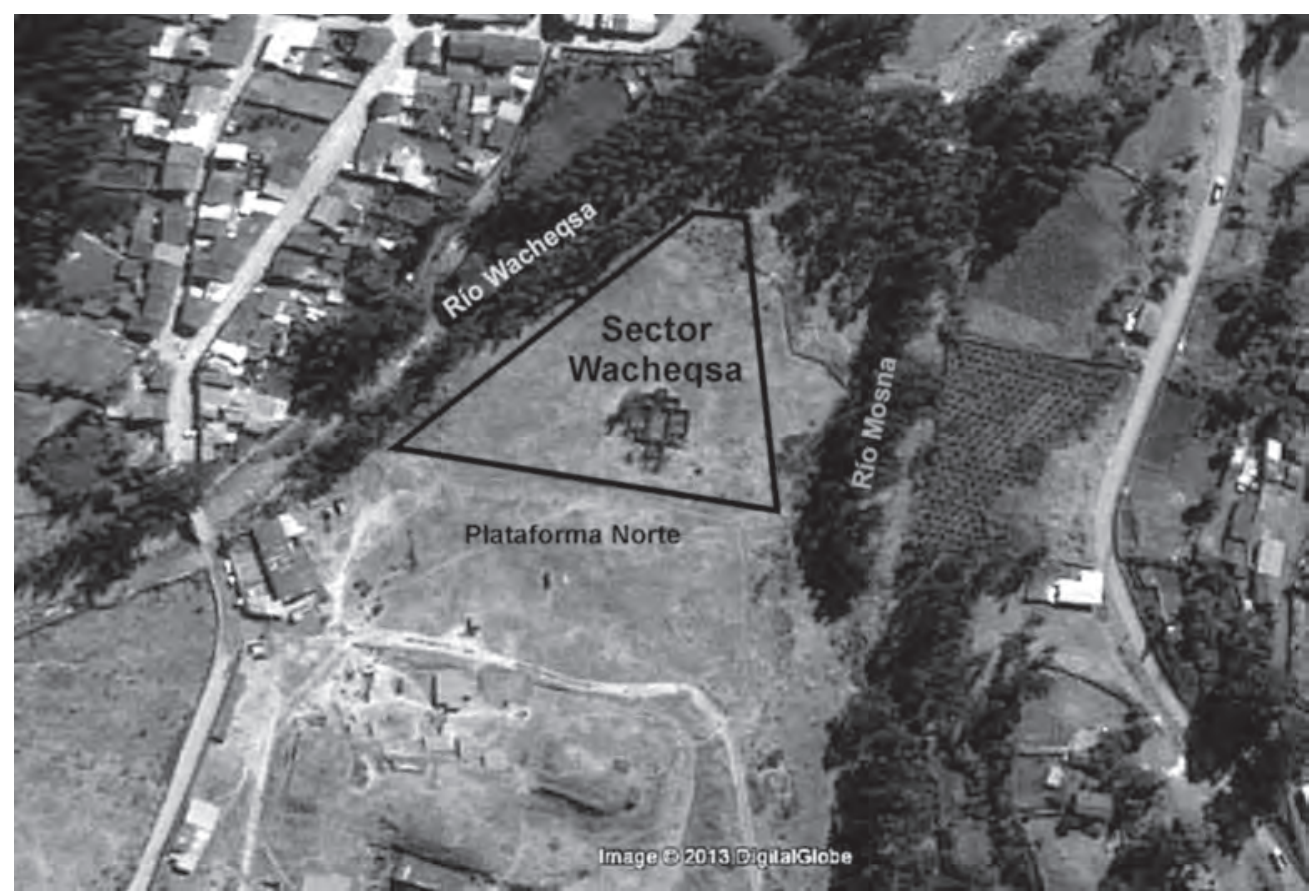

Figura 2. Ubicación del Sector Wacheqsa en Chavín de Huántar.

Mediante un cuidadoso programa de muestreo arqueológico y una larga, pero productiva, reconstrucción tridimensional estratigráfica, se han podido identificar cinco unidades espaciales prehistóricas (Fig. 3), que comprenden 700 años divididos en dos fases prehistóricas (Tabla 1).

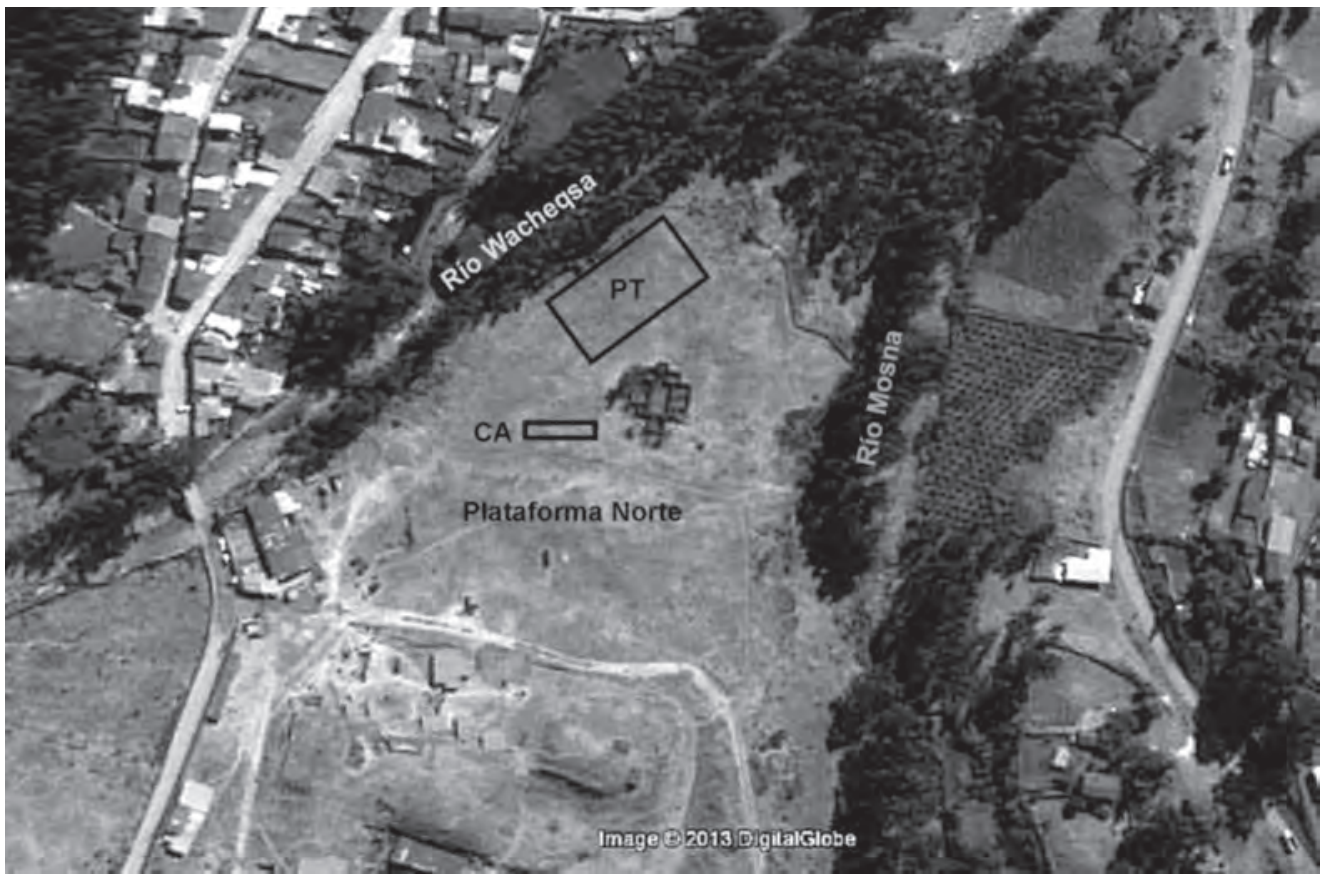

Figura 3. Fase del Formativo Medio en el Sector Wacheqsa. 
Tabla 01: Cuadro cronológico de Sector Wacheqsa

\begin{tabular}{|l|l|l|l|}
\hline Fechas & Fase & Unidades Espaciales & Ceramics \\
\hline & Moderna & Tierra agrícola, canal moderno, aluvión & \\
\hline 800-500 ANE & Formativo Tardío & Basural, Cuartos de Piedra, Plataformas Tardías & Janabarroide \\
\hline $1200-800$ ANE & Formativo Medio & Plataformas Tempranas, Correntera de Agua & Urabarroide \\
\hline
\end{tabular}

\section{DiVERSIDAD ESPACIAL EN EL SECTOR WACHEQSA}

La fase más antigua identificada en el sector Wacheqsa corresponde al Formativo Medio, el cual cubre los 1200-800 ANE (Mesia 2007, 2012; Rick et al. 2010). Está compuesta por las siguientes unidades espaciales: Correntera de Agua (CA) y Plataformas Tempranas (PT). CA está localizada en el extremo sur del sector Wacheqsa y comprende un área total estimada de $48 \mathrm{~m}^{2}$ y un volumen total estimado de $46 \mathrm{~m}^{3}$. La profundidad promedio de esta unidad espacial es de 3,05 $\mathrm{m}$ bajo superficie. Se caracterizó por una sucesión de niveles compactos de arena gris y verdosa alternados con grava fina y gruesa. Se excavaron en total $5,72 \mathrm{~m}^{3}$, recuperándose 330 elementos arqueológicos con una densidad promedio de 57 elementos por $\mathrm{m}^{3}$. La cerámica identificada en esta unidad se asemejan a las identificadas como Urabarriu por Burger (1984, 1998); no se encontró fragmento cerámico alguno relacionado o similar a formas o decoraciones janabarriodes. PT es una unidad localizada en las secciones norte y central del Sector Wacheqsa. Comprende un área total estimada de $1100 \mathrm{~m}^{2}$ y una profundidad promedio de 2,04 $\mathrm{m}$. Se caracteriza por una secuencia de depósitos con muy pequeña inclinación, con superficies compuestas por rocas angulares pequeñas y una baja densidad de materiales arqueológicos. Se excavó un total de $20,3 \mathrm{~m}^{3}$, recuperándose 978 elementos arqueológicos con una densidad promedio de 48 elementos por metro cúbico. Similar a CA, la cerámica recuperada en esta unidad se relaciona a la llamada Urabarriu (Fig. 4). La siguiente fase corresponde al Formativo Tardío, datándose entre los 800-500 ANE (Mesia 2007, 2012; Rick et al. 2010) (Fig. 5). Está compuesta por las siguientes unidades espaciales: Basural (B), Plataformas Tardías (PTA) y Cuartos de Piedra (CP). El Basural está localizado en el extremo sur del sector Wacheq-
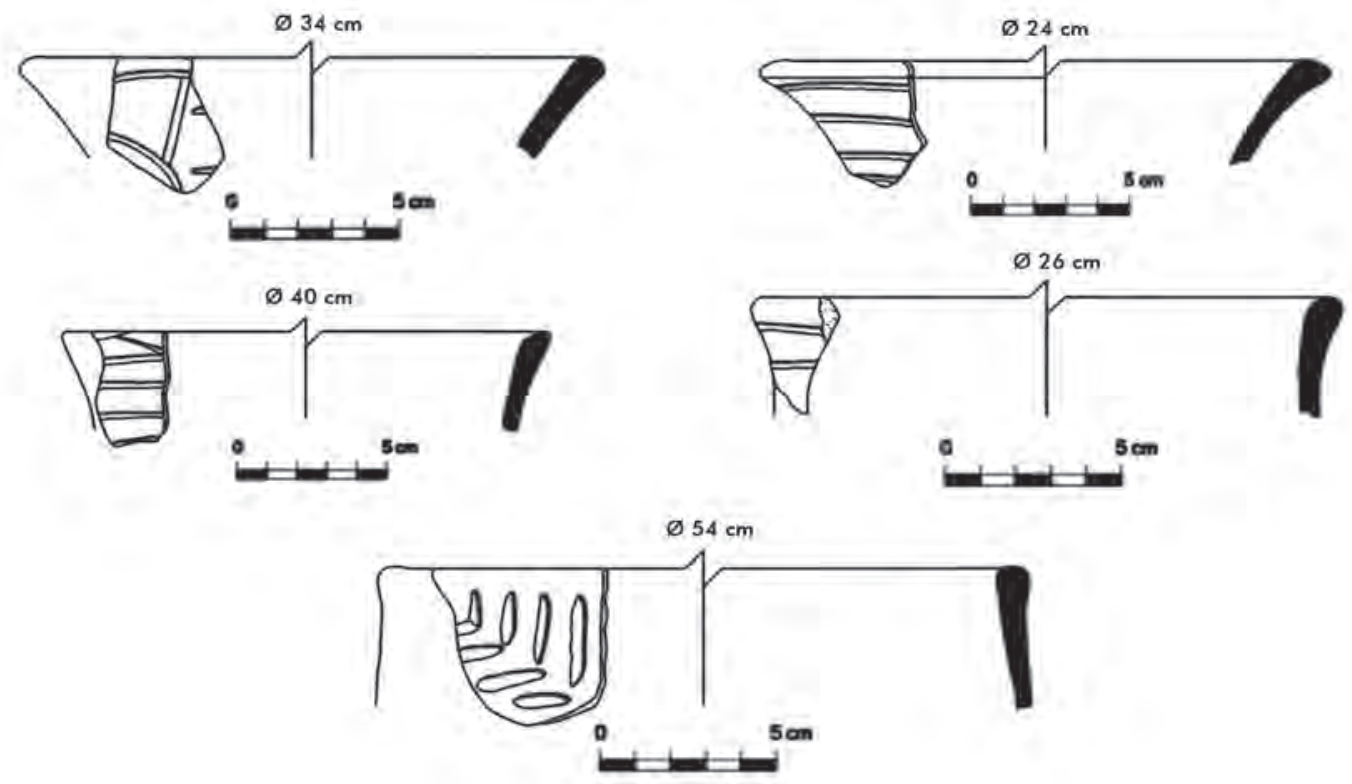

Figura 4. Cerámica del Formativo Medio en el Sector Wacheqsa. 


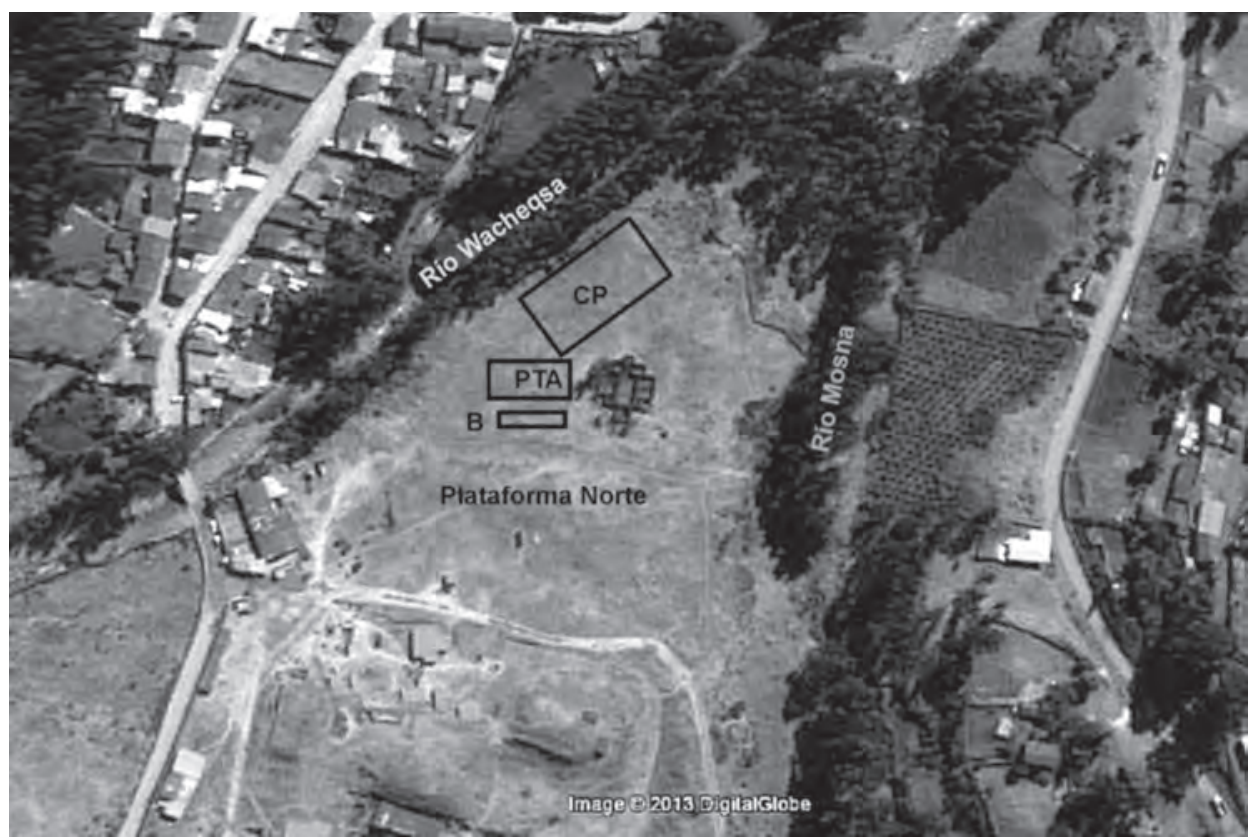

Figura 5. Fase del Formativo Tardío en el Sector Wacheqsa.

sa, sobre la unidad CA, comprendiendo un área total estimada de $48 \mathrm{~m}^{2}$, un volumen total estimado de $83 \mathrm{~m}$, con casi $2 \mathrm{~m}$ degrosor promedio. Se caracteriza por capas compactas y semicompactas mezcladas con rocas angulares y cantos rodados grandes y por una alta densidad de elementos arqueológicos. Se excavó un total de 22,03 $\mathrm{m}^{3}$, recuperándose 15814 elementos arqueológicos, obteniéndose una densidad general de 718,18 elementos por $\mathrm{m}^{3}$. La unidad PTA está localizada en la sección centro-sur del sector Wacheqsa y comprende un área estimada de $223 \mathrm{~m}^{2}$ con un volumen también estimado de $276 \mathrm{~m}^{3}$; se caracteriza por presentar una matriz compacta con abundantes rocas angulares y cantos rodados medianos y grandes, además de una baja densidad de elementos arqueológicos. Se excavó un total de 5,88 $\mathrm{m}^{2}$, recuperándose 102 elementos arqueológicos, obteniéndose una densidad de 17,3 elementos por metro cuadrado. La unidad CP está localizada en la sección central del sector Wacheqsa, directamente sobrela unidad PT, y comprende un área estimada de $1717 \mathrm{~m}^{2}$ y un volumen igualmente estimado de 668,47 $\mathrm{m}^{3}$. Presenta cuartos de piedra con pasajes interconectados, los cuales fueron cubiertos con un relleno suelto de cantos rodados y piedras angulares. Una vez que los cuartos y pasajes estuvieron cubiertos, se revistió la zona con una capa de piedras cuadrangulares de tamaño mediano, que formaron la superficie de extensa plataforma sobre los cuartos y pasillos. Se excavó un total de 22,3 $\mathrm{m}^{2}$ del que serecuperaron 3425 elementos arqueológicos, obteniéndose una densidad total de 154 elementos por metro cuadrado. La cerámica recuperada en esta fase es semejante a aquella identificada como Janabarriu por Burger $(1984,1998)$ (Fig. 6).

\section{ÍNDICE BOONE}

Para el presente análisis se utilizó información proveniente de 20649 materiales arqueológicos, provenientes de 200 depósitos estratigráficos, registrados en un área excavada de $132 \mathrm{~m}^{2}$ (0,9\% del sector Wacheqsa). Este alto nivel de complejidad estratigráfica impuso un reto interpretativo en la elucidación de la naturaleza del registro arqueológico, el modo en el cual estos componentes se relacionan uno con otro, así como la naturaleza de las actividades que originaron el registro materia del presente artículo. 


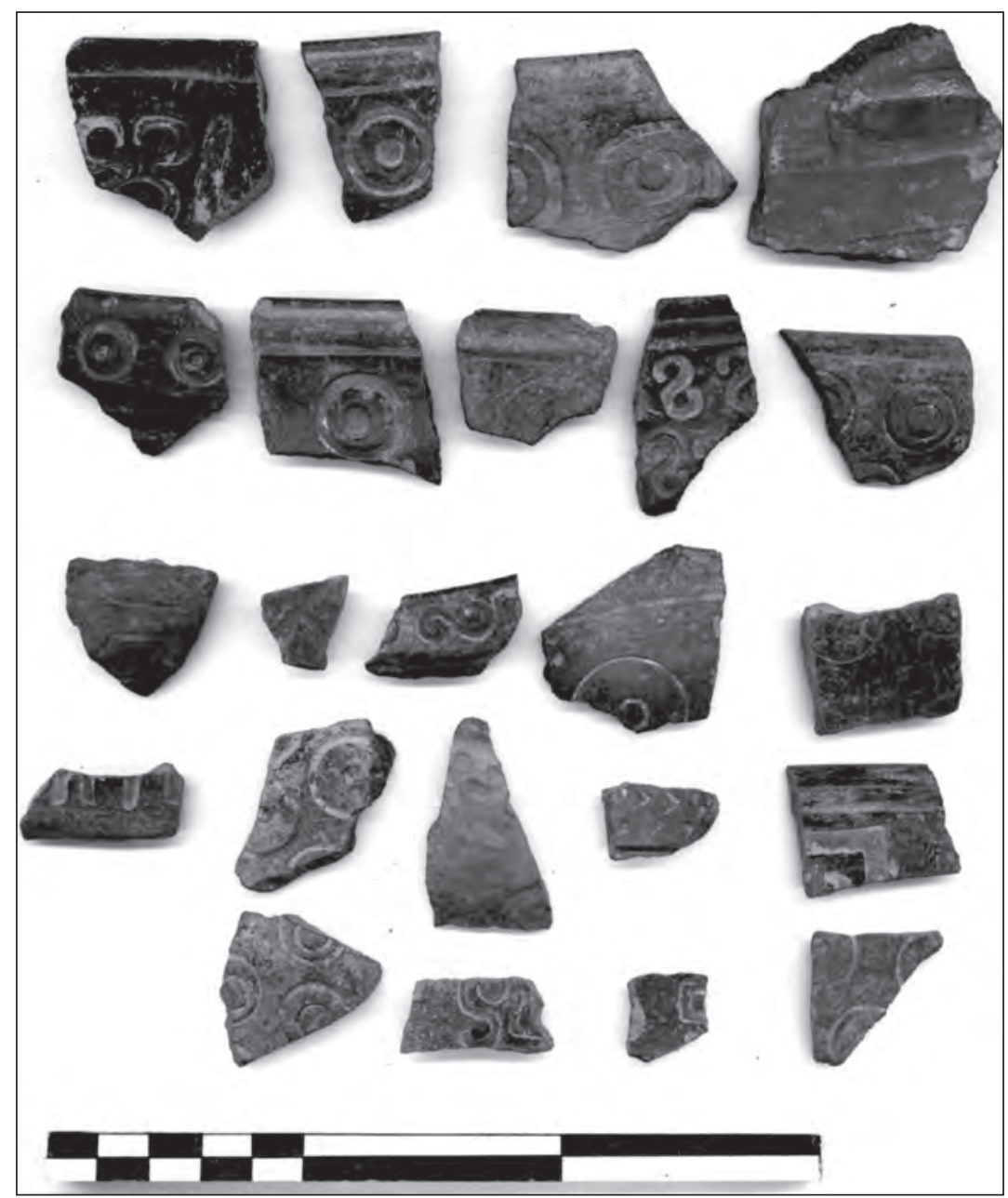

Figura 6. Cerámica del Formativo Tardío en el Sector Wacheqsa.

En primera instancia, de acuerdo a las unidades espaciales identificadas y mencionadas en el punto anterior, se estableció la distribución de densidad de artefactos arqueológicos por depósito estratigráfico y luego por unidad espacial. Esto debido a que la cuantificación de índices de densidad por cada clase de artefacto al interior de cada depósito, permite la caracterización de cada unidad espacial en términos de distribución de artefactos.

De acuerdo a las clases de materiales recuperados, las frecuencias de densidades fueron tabuladas a fin de identificar patrones de distribución por depósitos y unidades espaciales. Estas frecuencias fueron ingresadas al paquete estadístico JMP y contrastadas con cada unidad espacial a fin de identificar la variación en densidad existente entre los materiales por unidad espacial.

El índice Boone fue inicialmente concebido para el análisis comparativo de basurales (Boone 1987), sin embargo he adaptado el mismo a fin de poder utilizarlo con depósitos estratigráficos. De acuerdo a lo planteado por Boone, cada basural constituye una unidad de análisis, mientras que en presente trabajo, la unidad de análisis está compuesta por cada depósito estratigráfico. Boone cuantificó el número de artefactos y el número de clases de artefactos por basural mientras para el sector Wacheqsa se realizaron similares medidas pero por depósito estratigráfico. 
El propósito de este índice (Hi) es comparar las unidades de proveniencia individuales (depósitos) de artefactos con la distribución acumulada de todos los depósitos combinados. Valores elevados Hi indicarán un elevado grado de homogeneidad (prevalencia de una clase de artefactos sobre el resto de clases) e índices bajos de Hi, indicarán una baja homogeneidad y por consiguiente la heterogeneidad en la distribución de artefactos (Boone 1987). La variación espacial en la densidad ocupacional del asentamiento podría ser una razón obvia para una distribución no uniforme del tamaño de clases de artefactos al interior de los depósitos en un área dada. El tamaño de un depósito se refiere al número de artefactos presente en cada depósito.

Siguiendo lo propuesto por Boone, una perfecta heterogeneidad (valor Hi de 0) se alcanzará cuando todas las categorías definidas están presentes en cantidades iguales. La homogeneidad perfecta (valor 1 de Hi) se da cuando la población de artefactos existente está compuesta por una sola clase. Consecuentemente, al analizar los depósitos estratigráficos del sector Wacheqsa, una medida de heterogeneidad fue asumida, en la cual la mezcla de artefactos en la totalidad del sitio -la proporción relativa de artefactos en el sitio- es heterogénea en vista de que refleja la proporción relativa de acciones responsables de la producción de las actividades que produjeron los depósitos estratigráficos en un área dada (el sector Wacheqsa). De este modo se hace posible cuantificar los diferentes valores Hi entre los depósitos o incluso entre las unidades espaciales, agrupando todos los valores Hi de cada unidad espacial a fin de observar cómo se comportan una en relación a otra.

Este índice es calculado del siguiente modo:

* Los datos primarios están compuestos de frecuencias (yij) de una gama de clases de artefactos (j) las cuales son obtenidas de un número dado de depósitos (i). El número total de artefactos por cada clase se identifica como Yj.

* La expresión total de frecuencias relativas de todo el sitio se obtiene calculando la proporción de una clase total por cada clase restante. Esta relación se transforma en el factor de ponderación (Wj). Wj se calcula por cada clase de artefacto en cada depósito.

* Los porcentajes ponderados (pij) de cada clase en cada depósito son obtenidos dividiendo cada valor ponderado individual por la suma de todos los valores ponderados de un depósito dado.

* La diferencia entre 1 y el número de clases registrado $(j)$ es calculado.

* Finalmente, el índice Boone (Hi) se calcula en base a la suma de la diferencia al cuadrado de pij menos $\mathrm{Pj}$.

$$
\begin{aligned}
& W j=\frac{y i j}{Y j} \\
& \text { pij }=\frac{W j}{\Sigma W j} \\
& P j=\frac{1}{j} \\
& W j=\left[\Sigma(p i j-P j)^{2}\right]
\end{aligned}
$$

yij Frecuencia de clases de artefactos por depósito (número de ciertas clases de artefactos por depósito)

Yj Total de artefactos por clase en el sitio (número de total de ciertas clases de artefactos en todos los depósitos)

Wj Proporción total de una clase en relación al total de clases.

Pij Porcentaje ponderado de cada clase en cada depósito.

J Número de clases.

Los primeros dos pasos se siguieron de acuerdo a lo establecido por Boone (Boone 1987), mientras que los siguientes tres fueron sugerencias de Ian Robertson (Robertson 2007). Se consideraron 10 clases de artefactos a saber: fragmentos de cerámica decorada, fragmentos de cerámica diagnóstica, obsidiana, fragmentos de arcilla quemada, espejos de antracita, moluscos, líticos, puntas de proyectiles, artefactos de hueso y fragmentos de crisocola. La hipótesis a probar mediante el uso de este índice indica que todas las unidades espaciales están compuestas de depósitos que tienen valores Hi similares, es decir, que todos los depósitos estratigráficos de todas las unidades espaciales presentan proporciones similares de clases de materiales arqueológicos. 
Antes de evaluar los depósitos estratigráficos del sector Wacheqsa, es necesario indicar que «la relación entre tamaño de muestra y diversidad debe de ser investigada antes de cualquier interpretación» (Cruz-Uribe 1988: 194). El tamaño de una muestra puede afectar seriamente cualquier cuantificación de diversidad en tanto que depósitos extensos pueden tener mayor cantidad de clases (Boone 1987; Kintigh 1989; Orton 2000); además, dadas dos poblaciones con similar número de clases, una de las cuales presenta igual número de frecuencias para todas las clases, y la otra con altas frecuencias concentradas en un pequeño número de clases, los resultados indicarán índices menores de Hi para la primera población en comparación con la segunda. En tal sentido, para los cálculos del índice Boone en el presente trabajo, se ha diseñado un mecanismo de control del tamaño de la muestra.

Una vez que los valores Hi fueron calculados, se generó un intervalo de confidencia del 90\% a fin de identificar aquellos depósitos que debido a las restricciones impuestas por el tamaño de la muestra se encontraban fuera del intervalo de confidencia. En situaciones normales esto hubiera sido suficiente para controlar el modo en el cual el tamaño de la muestra afecta los resultados, sin embargo se decidió realizar varios análisis adicionales. Una vez que se generaron los valores Hi y sus intervalos de confidencia, se realizó un muestreo de la población analizada utilizando rutinas Monte Carlo a fin de determinar si los valores Hi calculados -incluso dentro de los intervalos de confidencia- podrían haber estado condicionados por el tamaño de la muestra. Las rutinas Monte Carlo son particularmente útiles al momento de probar la utilidad o validez de una rutina dada (en este caso del índice Boone), ya que «con un test de validez Monte Carlo sobre una prueba dada, la estadística es elucidada mediante la comparación de una muestra de la prueba, obtenida a través de la generación de muestras al azar utilizando un modelo particular» (Mainly 1991: 21. Citado por Shennan 2006: 64). Utilizando una rutina Monte Carlo, fue posible esclarecer si los valores Hi observados dentro y fuera del 90\% del intervalo de confidencia, representan un comportamiento real de los materiales arqueológicos al interior de los depósitos de las unidades espaciales identificadas o si tan solo eran un reflejo de las limitaciones del tamaño de la muestra.

De cualquier modo, fue importante verificar esta medida de diversidad a fin de clarificar la validez de su aplicación en el sector Wacheqsa. Todos los cálculos (índice Boone y rutinas Monte Carlo) fueron realizados utilizando el paquete estadístico $\mathrm{R}$.

\section{RESUlTADOS}

Como se describió previamente, este índice sirve para probar o descartar la hipótesis que indica lo siguiente: todas las unidades espaciales están compuestas por valores Hi similares. Se ha comparado las unidades de proveniencia individuales (depósitos estratigráficos) de artefactos con la distribución agregada de todos los depósitos combinados. Esta comparación ha permitido cuantificar la diversidad de todos los depósitos en relación con las clases de artefactos identificados. En tal sentido -como se indicó anteriormente- diez clases de artefactos han sido identificados, los cuales han sido promediados mediante el índice Boone.

Esta medida de diversidad permite observar si los depósitos de las unidades espaciales identificadas, forman agrupaciones sobre valores Hi particulares. En la figura $7^{1}$ se observan agrupaciones de valores Hi que en conjunto agrupan al 50\% de depósitos por unidad espacial. La unidad espacial con el valor Hi más bajo es el Basural, mientras que las Plataformas Tardías muestran el índice más alto. Las unidades Plataformas Tempranas y Correntera de Agua, casi se superponen íntegramente y muestran similares valores Hi, los cuales oscilan entre 0,2 y 0,85. La unidad Cuartos de Piedra está casi perfectamente agrupada alrededor de 0,4, superponiéndose parcialmente con el Basural. Una primera impresión indica una clara agrupación de valores HI por unidad espacial, sin embargo cabe

1 En los gráficos LP significa Plataformas Tardías; WF, Correntera de Agua; M, Basural; EP, Plataformas Tempranas; SR, Cuartos de Piedra. 
preguntarse ¿Cuánto de estas agrupaciones se deben al efecto del tamaño de la muestra? En la figura 8 se observa las diferencias entre unidad espacial de acuerdo al tamaño de la muestra de cada una. Si comparamos esta figura con la figura 9, observamos que la diferencia del tamaño de muestra de cada unidad espacial, es la que en realidad marca los valores Hi, ambos gráficos son idénticos. Las unidades espaciales con una muestra grande tendrán valores Hi bajos, siendo lo inverso para aquellas unidades con muestras pequeñas. Como se mencionó previamente, a fin de controlar este efecto, se calculó un intervalo de confidencia del 90\%, aquellos depósitos fuera de este intervalo supuestamente son anomalías al patrón esperado por el efecto del tamaño de la muestra dado por el intervalo e confidencia (figura 10). Este intervalo me dio los parámetros para considerar a aquellos depósitos fuera del mismo como outliers producto del efecto del tamaño de la muestra. Sin embargo, aún fue necesario corroborar que las agrupaciones de valores Hi por unidad espacial no se debieran al efecto del tamaño de la muestra. En tal sentido, se realizó un muestreo mediante una rutina Monte Carlo, realizando esta rutina 10000 veces a fin de obtener un patrón de variabilidad y descartar el mencionado efecto del tamaño de la muestra en los resultados. La figura 11 muestra la probabilidad de valores Hi ([p(Hi)] luego de realizar la rutina Monte Carlo, y se observa que la distribución de valores Hi es diferente a los observados en la figura 07, los valores Hi son diferentes a los p(Hi). Estos resultados confirman que los valores originales Hi eran producto del efecto del tamaño de la muestra, pero indican que en términos generales las unidades espaciales del sector Wacheqsa presentan altos índices de heterogeneidad. La figura 12 muestra la distribución de valores $\mathrm{p}(\mathrm{Hi})$ por unidad espacial. La media y la mediana general de $\mathrm{p}(\mathrm{Hi})$ es 0,225 y 0,1275 respectivamente.

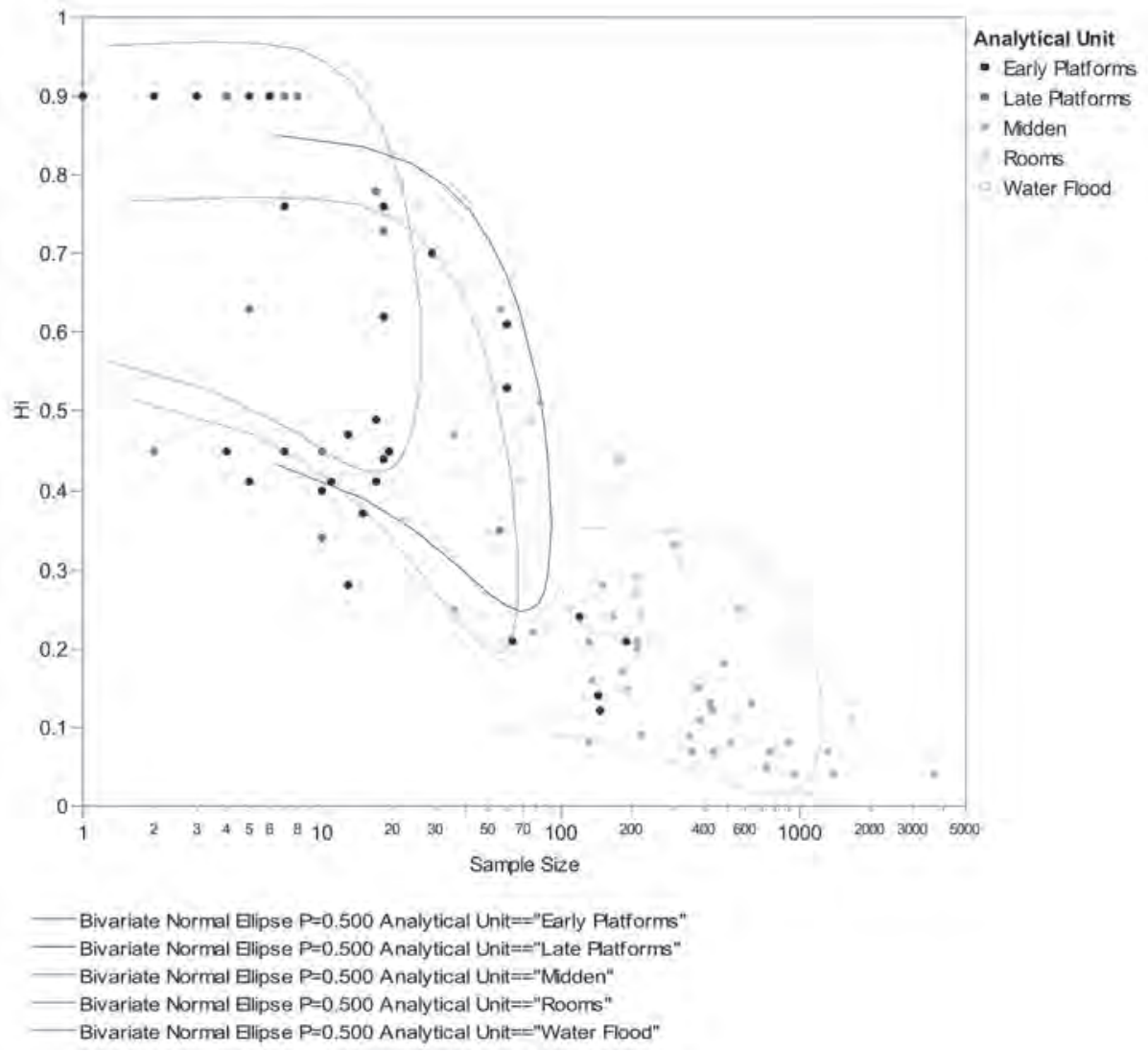

Figura 7. Índice Boone por unidad espacial. Nótese los agrupamientos de depósitos encerrados por líneas de colores. 


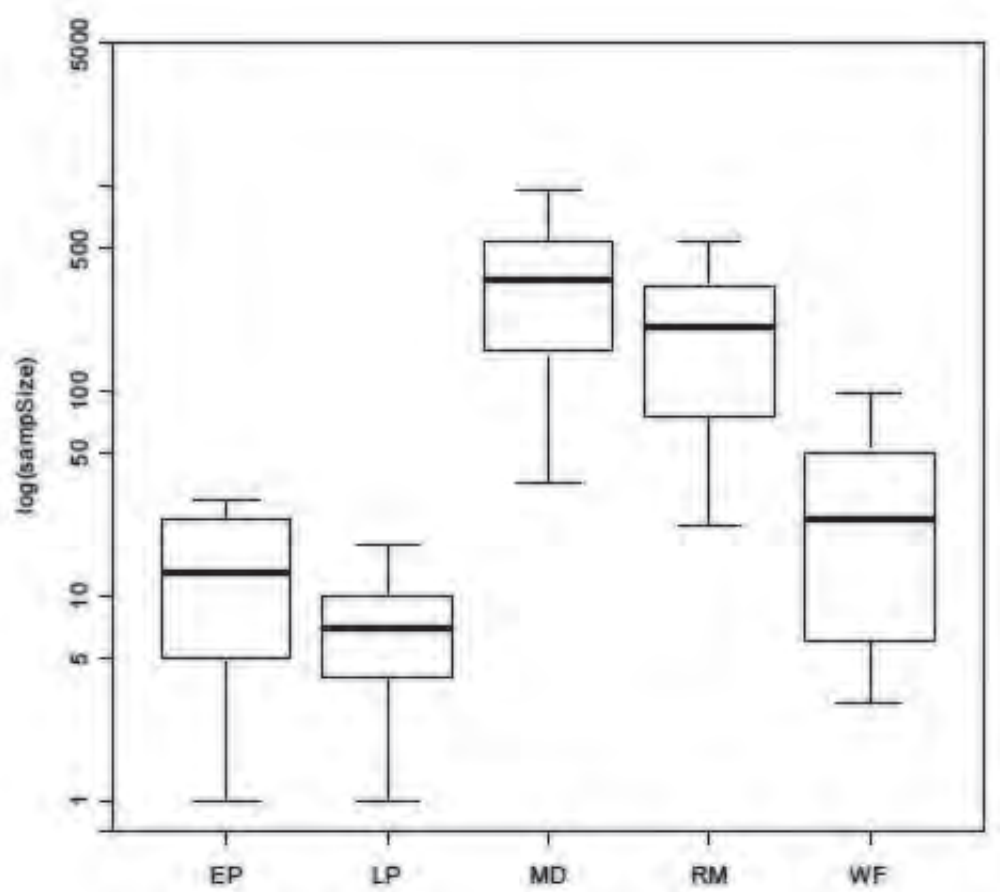

Figura 8. Distribución (log) de unidades espaciales de acuerdo al tamaño de muestra.

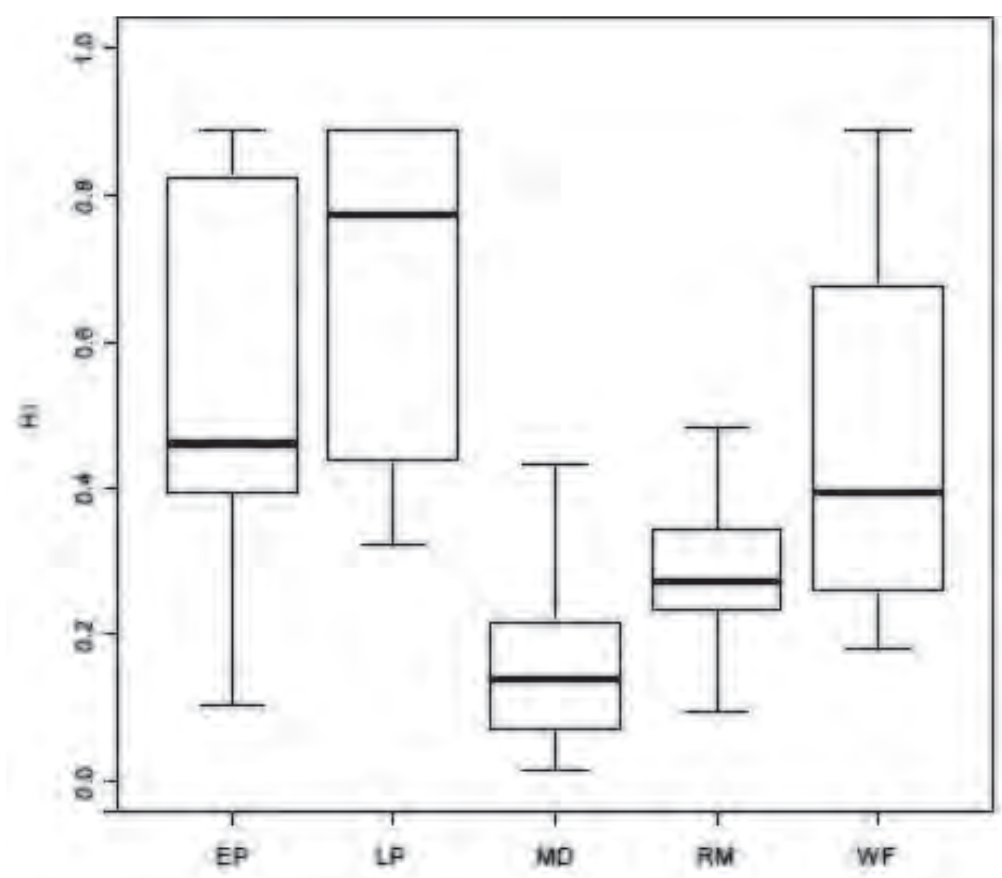

Figura 9. Distribución de unidades espaciales de acuerdo a valores Hi. 




Figura 10. Intervalo de confidencia (90\%) de valores $\mathrm{Hi}$.

La sección superior contiene el 85\% del intervalo, la sección inferior el 5\%.

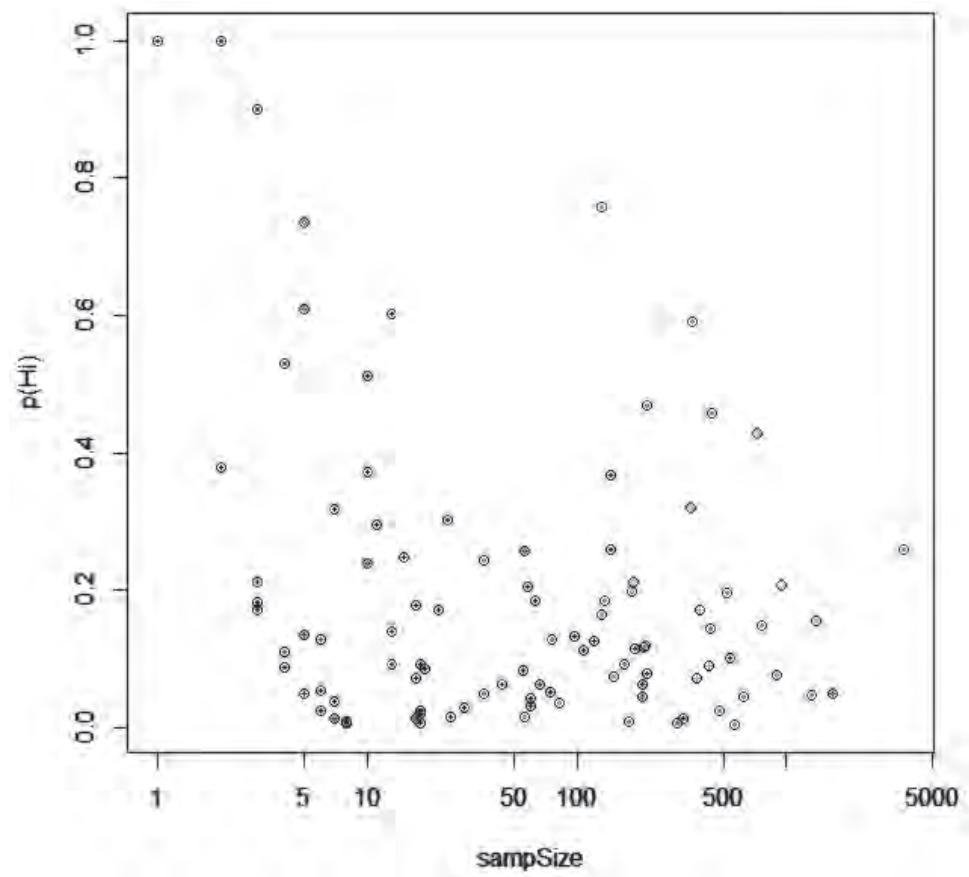

Figura 11. Probabilidades de valores $\mathrm{Hi}[\mathrm{p}(\mathrm{Hi})]$

luego de 10000 repeticiones mediante una rutina Monte Carlo. 


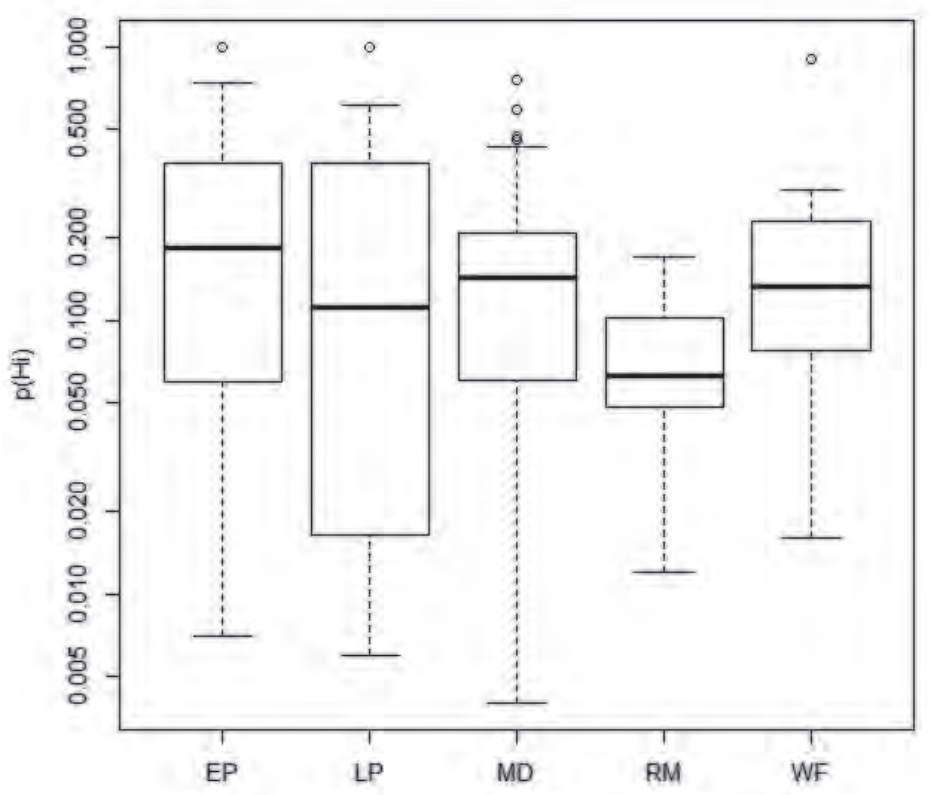

Figura 12. Distribución (log) de p (Hi) valores por unidad analítica.

\section{CONCLUSIONES}

Existen dos elementos que son necesarios de mencionar. El primero indica que las cinco unidades espaciales prehistóricas presentan valores bajos de $\mathrm{p}(\mathrm{Hi})$, y el segundo es que las unidades Cuartos de Pierda y Plataformas tempranas presentan valores significativamente diferentes de $\mathrm{p}(\mathrm{Hi})$, mientras que el resto de unidades espaciales se encuentran entre ambas, lo cual ha sido confirmado por una prueba $\mathrm{T}$ realizada sobre los valores $\mathrm{p}(\mathrm{Hi})$ de cada unidad espacial de acuerdo a lo que se observa en las tablas 02-04.

Tabla 02: Test $\mathrm{T}$ de la matriz del umbral de la diferencia menos significativa. Los valores positivos muestran pares de medias estadísticas significativamente diferentes

\begin{tabular}{|c|c|c|c|c|c|}
\hline & \multirow[b]{2}{*}{$t$} & \multirow{2}{*}{ Alpha } & & \\
\hline & & & & & \\
\hline & & 1,98667 & 0,05 & & \\
\hline Abs(Dif)-LSD & PTA & $\mathrm{CA}$ & PT & B & $\mathrm{CP}$ \\
\hline PTA & $-0,15680$ & $-0,09393$ & $-0,04499$ & $-0,02606$ & 0,03875 \\
\hline $\mathrm{CA}$ & $-0,09393$ & $-0,17689$ & $-0,12982$ & $-0,11148$ & $-0,04396$ \\
\hline PT & $-0,04499$ & $-0,12982$ & $-0,11734$ & $-0,09700$ & $-0,03820$ \\
\hline$B$ & $-0,02606$ & $-0,11148$ & $-0,09700$ & $-0,09917$ & $-0,04337$ \\
\hline$C P$ & 0,03875 & $-0,04396$ & $-0,03820$ & $-0,04337$ & $-0,18553$ \\
\hline
\end{tabular}

Tabla 03: Reporte de líneas de conexión del Test $\mathrm{T}$. Los niveles no conectados con la misma letra son significativamente diferentes

\begin{tabular}{|l|c|c|}
\hline \multicolumn{1}{|c|}{ Unidad Espacial } & Valor T & Media \\
\hline Plataformas tardías & $\mathrm{A}$ & 0,28521429 \\
\hline Correntera de agua & $\mathrm{AB}$ & 0,21200000 \\
\hline Plataformas tempranas & $\mathrm{AB}$ & 0,19172000 \\
\hline Basural & $\mathrm{AB}$ & 0,18008571 \\
\hline Cuartos de piedra & $\mathrm{B}$ & 0,07470000 \\
\hline
\end{tabular}


Christian Mesía / De lo cuantitativo a lo conductual: aplicaciones del índice Boone en el análisis...

Tabla 04: Test $\mathrm{T}$, reporte de diferencias

\begin{tabular}{|c|c|c|c|c|c|}
\hline Unidad Espacial & Unidad Espacial & Diferencia & CL Inferior & CL Superior & Valor p- \\
\hline Plataformas tardías & Cuartos de piedra & 0,2105143 & 0,038750 & 0,3822787 & 0,0168701 \\
\hline Correntera de agua & Cuartos de piedra & 0,1373000 & $-0,043961$ & 0,3185612 & 0,1358649 \\
\hline Plataformas tempranas & Cuartos de piedra & 0,1170200 & $-0,038203$ & 0,2722428 & 0,1377046 \\
\hline Basural & Cuartos de piedra & 0,1053857 & $-0,043367$ & 0,2541381 & 0,1627272 \\
\hline Plataformas tardías & Basural & 0,1051286 & $-0,026059$ & 0,2363158 & 0,1148805 \\
\hline Plataformas tardías & Plataformas tempranas & 0,0934943 & $-0,044987$ & 0,2319752 & 0,1832020 \\
\hline Plataformas tardías & Correntera de agua & 0,0732143 & $-0,093934$ & 0,2403623 & 0,3865037 \\
\hline Correntera de agua & Basural & 0,0319143 & $-0,111483$ & 0,1753113 & 0,6594398 \\
\hline Correntera de agua & Plataformas tempranas & 0,0202800 & $-0,129819$ & 0,1703785 & 0,7889888 \\
\hline Plataformas tempranas & Basural & 0,0116343 & $-0,096999$ & 0,1202676 & 0,8319903 \\
\hline
\end{tabular}

La unidad Cuartos de Piedra es la más heterogénea, mientras que la Plataformas Tardías la más homogénea. La hipótesis «todas las unidades espaciales están compuestas por valores Hi similares» es descartada, a pesar de que todos los promedios están bajo 0,3 (sugiriendo una alta heterogeneidad en sus contenidos), al interior de esta heterogeneidad existen diferencias entre las unidades espaciales, las cuales no se deben al efecto del tamaño de la muestra, sino a razones conductuales. Lo interesante es considerar que los resultados del índice Boone, indican que una sola actividad no fue la responsable de la producción de las cinco unidades espaciales prehistóricas identificadas, sino que cada una de ellas tuvo orígenes productivos, sociales, conductuales, etc., diferentes.

\section{Agradecimientos}

Los trabajos de excavación en Chavín de Huántar, que motivaron la investigación presentada en este artículo, fueron realizados con apoyo del Departamento de Ciencias Antropológicas de la Universidad de Stanford, el Centro de Estudios Latinoamericanos dela Universidad de Stanford, la Facultad de Humanidades de la Universidad de Stanford y la Fundación National Geographic.

Va un agradecimiento a Ian Robertson, quien fue crucial en el desarrollo del presente análisis estadístico al introducirme a la programación estadística y al uso de R, además de realizar valiosos comentarios al mismo. A John Rick por igualmente contribuir al desarrollo de la presente investigación y por su camaradería reflejada a lo largo de los años tanto en Stanford como en Chavín de Huántar. Igualmente a mis colegas y amigos con quienes he compartido discusiones extensas sobre Chavín de Huántar, la arqueología andina y otros tantos temas.

A los editores de Arqueología y Sociedad por publicar el presente estudio. Desde luego los errores incluidos en el presente trabajo son de exclusiva responsabilidad mía.

\section{BibliografíA}

BENNETT, Wendell

1944 The North Highlands of Peru, Excavations in the Callejon de Huaylas and Chavin de Huantar. Anthropological Papers of the American Museum of Natural History 39. The American Museum of Natural History, New York.

BOONE, James L., III

1987 Defining and Measuring Midden Catchment. American Antiquity 52(2): 336.

BURGER, Richard L.

1984 The Prehistoric Occupation at Chavín de Huántar, Perú. University of Calfornia Publications, Anthropology 14. University of Berkeley, Berkeley.

1988 Unity and Heterogeneity within the Chavin Horizon. In Peruvian Prehistory: An Overview of Pre-Inca and Inca Society, edited by R. Keatinge, pp. 99-144. Camabridge University Press, Cambridge. 
1998 Excavaciones en Chavin de Huantar. Pontificia Universidad Catolica del Peru, Lima.

CRUZ-URIBE, $\mathrm{K}$

1988 The use and meaning of species diversity and richness in archaeological faunas. Journal of Archaeological Science 15(2):179-196.

KEMBEL, Silvia

2001 Architectural sequence and Chronology at Chavin de Huantar, Peru. Ph D, Stanford University, Stanford.

2008 The Architecture at the Monumental Center of Chavín de Huántar: Sequence, Transfortmation and Chronology.Proceedings of the Chavin: Art, Architecture and Culture:35-84.

KEMBEL, Silvia and John RICK

2004 Building Authority at Chavín de Huántar: Models of Social Development in the Initial Period and Early Horizon. In Andean Archaeology, edited by H. Silverman, pp. 51-76. Blackwell Publishing, Oxford.

KINTIGH, Keith

1989 Sample Size, Significance, and Measures of Diversity. In Quantifying Diversity in Archaeology, edited by R. Leonard and G. Jones, pp. 25-36. Cambridge University Press, Cambridge.

LUMBRERAS, Luis

1977 Excavaciones en el Templo Antiguo de Chavín (Sector R). Informe de la Sexta Campaña. Ñawpa Pacha 15:1-38.

1989 Chavin de Huantar en el nacimiento de la civilizacion andina. Instituto de Estudios Andinas, Lima.

1993 Chavin de Huantar, excavaciones en la Galeria de las Ofrendas. KAVA, Mainz.

2007 Chavín: Excavaciones Arqueológicas I. Universidad Alas Peruanas, Lima.

MESIA, Christian

2007 Intrasite Spatial Organization at Chavín de Huántar during the Andean Formative: Three Dimensional Modeling, Stratigraphy and Ceramics, Anthropological Sciences, Stanford University, Stanford.

2012 Uso de Estimados de Densidad Kernel en la investigación de grupos cerámicos del periodo Formativo provenientes de Chavín de Huántar. Arqueología y Sociedad (24):161-190.

ORTON, C.

2000 Sampling in Archaeology. First ed. Cambridge University Press, Cambridge.

RICK, John

2005 The Evolution of Authority and Power at Chavin de Huantar, Peru. In Foundations of Power in the Prehispanic Andes, edited by D. O. a. C. A. C. Kevin J. Vaughn, pp. 71-89. vol. 14. American Anthropological Association, Los Angeles.

2006 Chavín de Huántar: Evidence for an Evolved Shamanism In Mesas and Cosmologies in the Central Andes, edited by D. Sharon. vol. 44. San Diego Museum, San Diego.

2008 Context, Construction, and Ritual in the Development of Authority at Chavín de Huantar. In Chavin; Art, Architecture and Culture, edited by W. Conklin and J. Quilter, pp. 3-34. Cotsen Institute of Archaeology, Los Angeles.

RICK, J. et al.

2010 «La cronología de Chavín de Huántar y sus implicancias para el Período Formativo». En: Boletín de Arqueología PUCP, Nº13:87-132. Lima: Pontificia Universidad Católica del Perú.

Robertson, Ian

2007 Personal communication.

SHENNAN, S.

2006 Quantifying Archaeology. Second ed. University of Iowa Press, Iowa.

TELLO, Julio C.

1960 Chavin Cultura Matriz de la Civilizacion Andina. Universidad Nacional Mayor de San Marcos, Lima. 